\title{
A Lifecycle Data Management System Based on RFID Technology of EPC Class1 Gen2 v2
}

\author{
Young-woo Kim and Jinwoo Park ${ }^{*}$ \\ Department of Industrial Engineering/Automation System Research Institute (ASRI), \\ Seoul National University, Seoul, Republic of Korea \\ ywkim@mailab.snu.ac.kr, autofact@snu.ac.kr
}

\begin{abstract}
To cope with pollution, exhaustion of resources and new regulations extending producer responsibility, the original equipment manufacturers are working hard towards an efficient solution that solves those problems. We propose an RFID (Radio Frequency IDentification) based information system which incorporates large user memory banks of EPCC1G2 v2 standard to follow up lifecycle information about the product. We use hierarchical data models to minimize storage requirement while storing all information about the whole product structure including subassembly and end components. Later we can extract the information to utilize maintenance histories and chemical structure of each component. We also consider applicability of our system in XML hierarchical data structure for the possibility of internet usage.
\end{abstract}

Keywords: Remanufacturing, Data System, Radio Frequency Identification, Closed-loop Supply Chain Management, EPCC1G2 (EPC Class 1 Generation 2).

\section{Introduction}

As time goes on, environmental problems such as climate change, destruction of ozone layer and depletion of natural resources are getting worse. For the environmental problems, several movements for saving the environments have been taken place both globally and locally. From a standpoint of manufacturing, product recycling is the way to take part in the environmental movements.

Also the development of brand-new materials as a substitute for natural resources could be the solution for preventing the depletion of natural resources, but it couldn't substitute perfectly. So collecting the products in end-of-life (EOL) phase and recovering the returned products as resources could reduce virgin sourcing obviously. Then the product recycling is considered the most active solution.

Product recycling could give an environmental advantage of extending lifespan of natural resources minded before, as well as an economic advantage from reducing the cost caused by virgin sourcing.

But when the manufacturers deal with a problem of product recycling, quality of returned product may vary significantly according to the age or history and environ-

\footnotetext{
* Corresponding author.

B. Grabot et al. (Eds.): APMS 2014, Part II, IFIP AICT 439, pp. 294-301, 2014.

(C) IFIP International Federation for Information Processing 2014
} 
ment of usage. Nowadays many products consist of many components and subassemblies. But they may have all different ages and expected lives. For example, a new component whose expected life is 20 years can be used more than once if it was used as a maintenance part for a product whose remaining expected life is, say, 5 years. In that sense if the life history of components and products are not available, recyclable components in good condition may be disposed after being recycled only once.

According to Fleischmann (2001) and Thierry et al. (1995), there are uncertainties in terms of timing, quantity and quality of product return flows[1][2]. And Kulkarni et al. (2005) describes the importance of information that would be helpful to handle the product disposition decisions on the most appropriate recovery option according to the condition of the returned products - the product can be reused as is, reused after remanufacturing, recycled to recover its material contents or incinerated for thermal energy recovery - and product recovery decisions by inventory visibility [3].

To handle the uncertainty problems, we propose a framework for the lifecycle data management system which gives a unique identifier to as many components and subassemblies as, and tracks historical events such as repair logs and useful data to solve the product disposition decision by tagging in chemical composition information.

To that end, we focus on Electronic Product Code Class1 Gen2 (EPCC1G2) v2 RFID technology because this new standard is shows the way to be adopted by all standard bodies including ISO and if offers a large user bank memory [3].

\section{A Framework for the Lifecycle Data Management System}

RFID has been considered as an enabler to launch the lifecycle data management system in many researches. Bajic and Chaxel (2002) concerned the use of automatic identification devices (Auto-ID tags) as permanent escort memories associated to the car during its whole life cycle. These memories provide an integration frame for vehicle lifecycle information, which are necessary to support the manufacturing, aftersales services and recycling processes [4]. Jun et al. (2009) proposed an overall framework for RFID applications in product lifecycle management with a Product Embedded Information Device (PEID) [5]. And Parlikad and McFarlane (2007) showed qualitatively that the availability of product information has a positive impact on product recovery decisions, and discuss how RFID-based product identification technologies can be employed to provide the necessary information [6].

RFID also could help operating an efficient supply network. It promises to eliminate manual inventory counting, warehouse mis-picking, and order-numbering mistakes by providing precise data on product location, product characteristics, and product inventory levels [7]. Also RFID traceability gives several advantages for enterprises to effectively handle their daily operations with distribution transparency [8]. Networked RFID systems can provide an automated and efficient approach for capturing and delivering complete item level product information in an accurate and timely manner thereby making it possible for high level decisions and process improvements during product recovery stages [3]. 


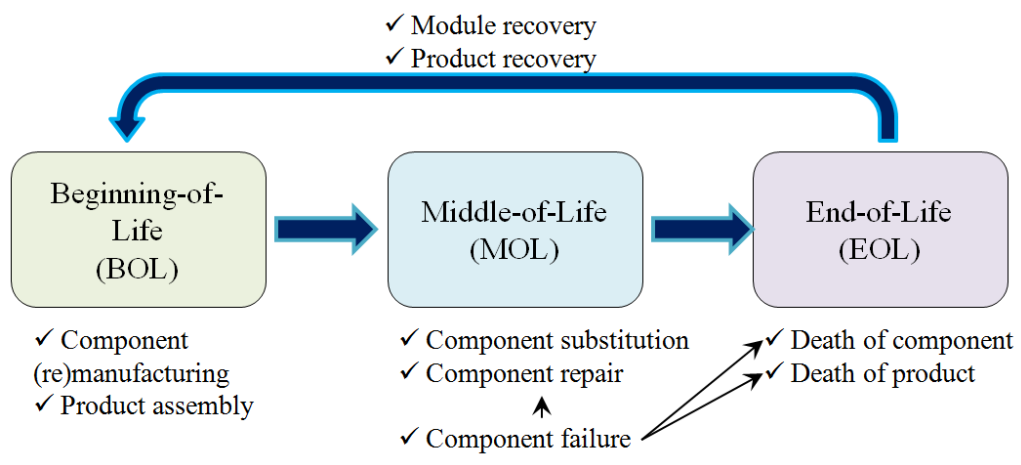

Fig. 1. Events occurred over whole lifecycle

Every data over the lifecycle as shown in Fig. 1 can be stored by combining external databases and the RFID tags attached to a product. The external databases consist of information about every product produced by Original Equipment Manufacturers (OEMs) and every component the OEM produces.

At the beginning-of-life (BOL) phase, products and its components acquire their own identifiers according to the data standard claimed by EPCglobal. EPC is a wellknown universal identification system for any physical object and it is considered as the most important data standard for supply chain applications. So it is widely used in informations systems that need to track or otherwise refer to physical objects with RFID tags.

A product could fail during its MOL (middle-of-life) phase. Product failure is caused by single or multiple component failures. At this point, a product user should decide to repair/substitute failed parts or dispose the product. If the user decide to repair the failed component and use the product continuously, the failed product still remains in the MOL phase. Information about the failed component should be written to the history $\log$ in the RFID tag attached to the repaired component. Also the product may still enter again into the MOL phase if the user decide to change failed parts to new ones. For this substitution case, only the failed component enter into EOL phase and collected by a 3rd party reprocessing facility or the OEM who is responsible for extended producer responsibility (EPR). EPR is the preventive conceptual strategy to save environment that was quoted first in the report of Swedish government [9] and EPR claims that every OEM takes responsibility to collect, recover, and reuse obsolete products and dispose on their own [10].

For the final option to cope with product failure, the user could decide to dispose the failed product if the repair cost is not worth by repairing or changing the failed component(s).

Failed components itself or components from failed products are collected and they could be recycled as follows.

- Reused after treatment

- Recovered as resources after dismantle, shredding, and additional treatments

- Recovered as thermal energy by incineration 
A reprocessing facility should make a recycling decisions among the various options described above by considering visual conditions, expert opinions and history logbooks in the attached tag.

All the returned products and components are came into the remanufacturing shop floor and booked into the inventory database of the OEM. Once the returned products and components are booked in, a preliminary sorting is performed to filter out components that do not apparently have much market value. In this step, automated identification and history logs of returned components about repair logs could be helpful to the experts who filter out the returned components in a bad condition.

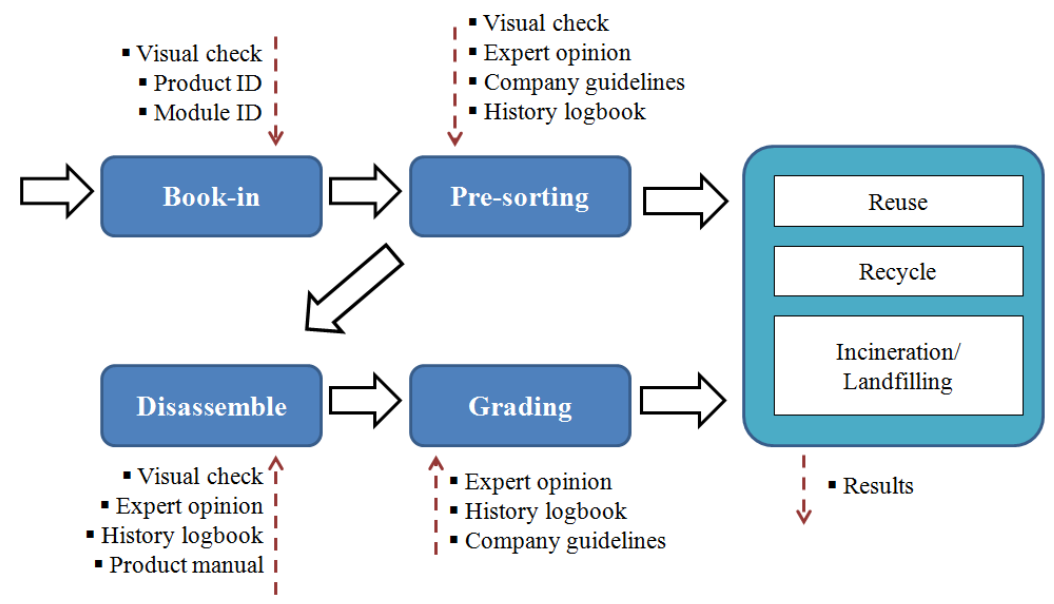

Fig. 2. Reverse process on the remanufacturing shop floor

Also the OEM can calculate environmental and economical effect both caused by different option taken. It is helpful to make more efficient decision while obeying the environmental regulations.

Also after the recovery process ended, results of recovery option actually taken by OEM's decision will be stored in a external database. From an administrative point of view, it could be an obvious clue for penalties incurred to OEMs who don't obey the regulations or for incentives to OEMs showing excellent recycling performance.

\section{Proposed System for Applying EPCC1G2 v2}

EPCC1G2 is the standard working framework proposed by GS1 in 2006 and is widely used for identification in the ultrahigh frequency (UHF) band. It has been a truly unique international protocol with faster read speed and more powerful security.

And the latest released air interface protocol, EPCC1G2v2, is an answer by GS1 to the requirements of the EPCglobal user community. The most impressive feature is enhanced user memory for alteration electronic article surveillance and supplementary encodings such as maintenance logging [12]. Therefore, EPCC1G2v2 technology seems to be very appropriate for sub-level tagging and launching of the proposed system. 
Table 1. Difference between Gen1 and Gen2 [11]

\begin{tabular}{|c|c|c|c|}
\hline & \multicolumn{2}{|c|}{ Gen1 } & Gen2 \\
\hline Standard & \multicolumn{2}{|c|}{4 UHF standards } & Unique international protocol \\
\hline Reader mode & \multicolumn{2}{|c|}{ Single-reader mode } & Single/Multi-reader mode \\
\hline Session & \multicolumn{2}{|l|}{$\mathrm{X}$} & $\underline{\mathrm{O}}$ \\
\hline \multirow{2}{*}{ Tag memory } & Class 0 & WORM * & \multirow{2}{*}{ WMRM ** } \\
\hline & Class 1 & WORM & \\
\hline \multirow{2}{*}{$\begin{array}{l}\text { Data transmission } \\
\text { speed }\end{array}$} & Class 0 & $80 \mathrm{~kb} / \mathrm{s}$ & \multirow{2}{*}{$\underline{640 \mathrm{~kb} / \mathrm{s}}$} \\
\hline & Class 1 & $140 \mathrm{~kb} / \mathrm{s}$ & \\
\hline \multirow{2}{*}{$\begin{array}{l}\text { Length of } \\
\text { password }\end{array}$} & Class 0 & 24 bit & \multirow{2}{*}{$\underline{32 \text { bit }}$} \\
\hline & Class 1 & 8 bit & \\
\hline
\end{tabular}

* WORM: Write Once Read Many ** WMRM: Write Many Read Many

We will discuss a data standard which specifies what is contained in the memory portion of the RFID tags, as well as the format. EPCC1G2 standard, also known as ISO18000-6C specifies the protocols that are used for communication between tags and readers as well as data structure requirements which are compatible with the EPC.

Table 1. GRAI-170 EPC scheme [11]

\begin{tabular}{|c|c|c|c|c|c|}
\hline Header & Filter Value & Partition & $\begin{array}{c}\text { Company } \\
\text { Prefix }\end{array}$ & Asset Type & $\begin{array}{c}\text { Serial } \\
\text { Number }\end{array}$ \\
\hline 8 bits & 3 bits & 3 bits & $20 \sim 40$ bits & $24 \sim 4$ bits & 112 bits \\
\hline
\end{tabular}

EPCglobal officially announced several data standards for different usage. Using a data standard in existence could pursue interoperability then we could expect enhancing applicability for worldwide usage. We focus on the GRAI (Global Returnable Asset Identifier) standard which is typically used for a specific returnable asset such as reusable shipping containers, pallets because we consider the EOL products and components as resources. Then we select the GRAI-170 EPC tag data standard because memory space of 170 bits seems to be enough space to assign every product and component. Basically we could identify at most $2^{112}$ individual components with same type by using the GRAI-170 EPC scheme. A company that use GRAI applications could take advantage of identifying the returnable asset ownership for the life of the asset wherever it goes, facilitating cross company asset management tracking, guarding against loss [13]. And also the standard scheme could be adjustable for memory portion by setting different partition value to reflect characteristics of target product's BOM. If the number of companies associated with a target industry is relatively low and the number of components composed in products is relatively high because the products manufactured are somewhat complex, we could assign smaller space to company prefix while assign larger space to asset type. 


\begin{tabular}{|c|c|c|c|c|c|c|c|c|}
\hline Bank & \multicolumn{3}{|c|}{ Kill Password } & \multicolumn{5}{|c|}{ Access Password } \\
\hline \multirow[t]{2}{*}{$\begin{array}{r}\text { Bank } \\
01\end{array}$} & & $\cdots$ & Header & F.V. & Partition & C.P & AssetType & $\underline{\text { Serial }}$ \\
\hline & & & \multicolumn{6}{|c|}{ GRAI-170 EPC scheme } \\
\hline $\begin{array}{r}\text { Bank } \\
10\end{array}$ & \multicolumn{8}{|c|}{ TID memory } \\
\hline $\begin{array}{l}\text { Bank } 11 \\
\text { (User }\end{array}$ & $\cdots$ & DeleteBit & Paren & & History & $\log$ & Instruc & ons \\
\hline
\end{tabular}

Fig. 3. Conceptual design of EPCC1G2v2 tags

EPCC1G2v2 tags are logically divided into 4 banks with user memory space. So we also analyze an encoding scheme to pack useful data efficiently. In this context, there are two major problems around the encoding issue: We should figure out what kind of data should be contained in the tag, and how to encode data economically.

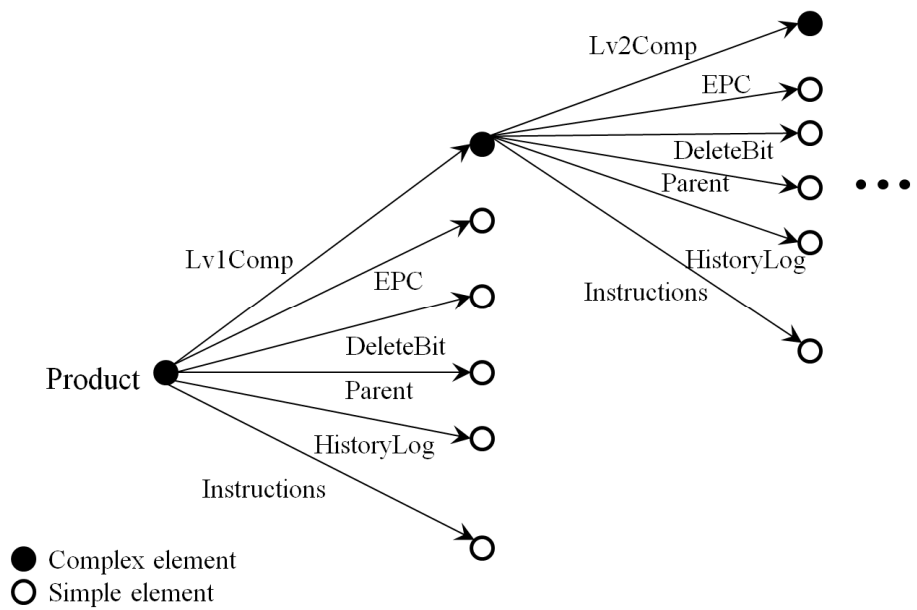

Fig. 4. Hierarchical data representation

We develop a data model taking into account of both the IMS(Information Management System) structure of the old IBM database technology [14] and the XML (eXtensive Markup Language) hierarchical data model for encoding user memory space. XML provides information about the structure and meaning of the data in the Web pages rather than just specifying how the Web pages are formatted for displaying on the screen. So XML has emerged as the standard for structuring and exchanging data over the Web. The XML could represent hierarchical data by possessing relatively smaller space then it could be a solution for tag sizing and reflecting perfectly modular structure because a bill-of-material of any modular product takes the form of hierarchical structure. 
Even if history logs and instructions used in recovery process don't have any data structure, XML hierarchical data structure could be a good encoding scheme because it supports writing semi-structured documents. However, it seems to perform better if the data structure for history logs and instructions are well-defined such as the WEEE-vector [15]. The hierarchical model could be transformed tree structure into graph E-R (Entity-Relationship) model if the referential integrity constraint is satisfied. Then we could simply mapping of the hierarchical structure to popular relational model.

\section{Conclusion}

We propose the RFID-based modular lifecycle data management system with component-level RF tagging to track and manage event log data of products and its components over whole lifecycle. Obtained lifecycle data could handle the quality uncertainty problem then also it could be helpful to take the most appropriate recovery option to maximize resource utilization by multiple recovery. And it could relieve workload and reduce cost of sorting process because the incremental cost of each scan is very low by automated identification.

From an manufacturers point of view, the optimal production planning with considering remanufactured components could be enabled by increasing inventory visibility. And the proposed system could help environmental administrators by playing the role of correct monitoring system from an administration point of view because real time data gathered couldn't be manipulated.

We consider the advantages when the EPCC1G2v2 standard protocol is applied to the reverse logistics process. EPCC1G2v2 promises more space to store user-defined data in user memory bank. If useful information is provided during recycling process on the shop floor, the workers involved in pre-sorting and grading of returned components could use the information to make the most appropriate decision in order to maximize resource utilization about recovery options immediately without external data access. Also we examine the potentiality of using GRAI-170 EPC scheme as the data standard. Its multidimensional encoding scheme with filter value, partition value and asset type promises enough space to tag every component in the target industry.

Also we introduce the XML hierarchical data model as the solution for RF tag data encoding. It would save the memory space by minimizing memory possession that is the main advantage of using hierarchical structure. It also easily transformed into relational data structure widely used.

Obeying current EPC standards promises great interoperability and we hope this work could be the first step to building a centralized monitoring/management system with EPC discovery service.

And structuring of history logs and instructions should be followed up to make perfect structured data-centric XML documents. It would be found out in our following working paper. 


\section{References}

1. Fleischmann, M.: Quantitative Models for Reverse Logistics. Lecture Notes on Economical and Mathematical Systems, vol. 501. Springer (2001)

2. Thierry, M., Salomon, M., van Nunen, J., van Wassenhove, L.: Strategic Issues in Product Recovery Management. California Management Review 37, 114-135 (1995)

3. Kulkarni, A., Parlikad, A., McFarlane, D., Harrison, M.: Networked RFID Systems in Product Recovery Management. In: Proceedings of the 2005 IEEE International Symposium on Electronics and the Environment, pp. 66-71 (2005)

4. Bajic, E., Chaxel, F.: Auto-ID Mobile Information System for Vehicle Life Cycle Data Management. In: Proceedings of the IEEE Conference on Systems, Man and Cybernetics (2002)

5. Jun, H., Shin, J., Kim, Y., Kiritsis, D., Xirouchakis, P.: A Framework for RFID Applications is Product Lifecycle Management. International Journal of Computer Integrated Manufacturing 22, 595-615 (2009)

6. Parlikad, A., McFarlane, D.: RFID-based Product Information in End-of-Life Decision Making. Control Engineering Practice 15, 1348-1363 (2007)

7. Niederman, F., Mathieu, R., Morley, R., Kwon, I.: Examining RFID Applications in Supply Chain Management. Communications of the ACM 50, 93-101 (2007)

8. Lee, D., Park, J.: RFID-based traceability in the supply chain. Industrial Management and Data Systems 108, 713-725 (2008)

9. Lindhqvist, T., Lidgren, K.: Model for Extended Producer Responsibility: In Ministry of the Environment. From the Cradle to the Grave - six studies of the environmental impacts of products, pp. 7-44. Ministry of Environment, Stockholm (1990)

10. Lindhqvist, T.: Extended Producer Responsibility in Cleaner Production. IIEEE Dissertatons, Lund University (2000)

11. GS1, EPC Radio-Frequency Identity Protocols Generation-2 UHF RFID Version 2.0.0 Ratified (2013)

12. Martin, H., San Millan, E., Peris-Lopez, P., Tapiador, J.: Efficient ASIC Implementation and Analysis of Two EPC-C1G2 RFID Authentication Protocols. IEEE Sensors Journal 13, 3537-3547 (2013)

13. GS1, GS1 Identification Key Series - GRAI (Global Returnable Asset Identifier) Issue 1.1 (2008)

14. Date, C.: An Introduction to Database Systems, 5th edn., vol. I. Addison-Wesley Publishing Company (1990)

15. Luttropp, C., Johansson, J.: Improved Recycling with Lifecycle Information Tagged to the Product. Journal of Cleaner Production 18, 346-354 (2010) 